\title{
Gas Phase Electrodeposition: A Programmable Multimaterial Deposition Method for Combinatorial Nanostructured Device Discovery
}

\author{
En-Chiang Lin, Jesse J. Cole, and Heiko O. Jacobs* \\ Electrical Engineering, University of Minnesota, Room 4-178, 200 Union Street SE, Minneapolis, \\ Minnesota 55455, United States
}

\begin{abstract}
This article reports and applies a recently discovered programmable multimaterial deposition process to the formation and combinatorial improvement of 3D nanostructured devices. The gas-phase deposition process produces charged $<5 \mathrm{~nm}$ particles of silver, tungsten, and platinum and uses externally biased electrodes to control the material flux and to turn deposition ON/OFF in selected domains. Domains host nanostructured dielectrics to define arrays of electrodynamic $10 \times$ nanolenses to further control the flux to form $<100 \mathrm{~nm}$ resolution deposits. The unique feature of the process is that material type, amount, and sequence can be altered from one domain to the next leading to different types of nanostructures including multimaterial bridges, interconnects, or nanowire arrays with $20 \mathrm{~nm}$ positional accuracy. These features enable combinatorial nanostructured materials and device discovery. As a first demonstration, we produce and identify in a combinatorial way 3D nanostructured electrode designs that improve light scattering, absorption, and minority carrier extraction of bulk heterojunction photovoltaic cells. Photovoltaic cells from domains with long and dense nanowire arrays improve the relative power conversion efficiency by $47 \%$ when compared to flat domains on the same substrate.
\end{abstract}

KEYWORDS Programmable electrodeposition, arc discharge, atmospheric pressure gas phase deposition, nanoparticle nanostructured electrodes, combinatory device discovery, printable flexible electronics, plasmas and aerosols

I norganic nanomaterials in the form of nanoparticles and nanowires have attracted attention due to their unusual properties including quantum size effects, high surface area-to-volume ratio, and increased catalytic activity. These properties have influenced almost every research discipline including the fields of printable electronics, optoelectronics, physical sensors, photovoltaics, biological screening, and environmental protection. ${ }^{1-5}$ Available synthesis techniques can be grouped into two classes: low-temperature wet chemical and high-temperature gas/vapor phase methods. Gas/vapor phase methods such as thermal flow synthesis, ${ }^{6-8}$ laser ablation, ${ }^{9}$ sputtering, ${ }^{10}$ plasma induced synthesis, ${ }^{11}$ and arc discharge ${ }^{12,13}$ can produce nanoparticles and nanowires of various types including high-temperature and highperformance semiconductors that are difficult to make using low-temperature wet chemical methods.

A challenge today can be found in the design of gas phase systems that combine high-temperature synthesis with lowtemperature local area deposition on foreign substrates for printable electronics applications. Selected area deposition schemes are well established in the liquid phase but remain largely absent in the gas phase. Current direct write gasphase deposition methods use nozzles, or aerodynamic

* To whom correspondence should be addressed. E-mail: hjacobs@umn.edu Received for review: 07/05/2010

Published on Web: 10/14/2010 beams ${ }^{14,15}$ to deposit the materials locally in a serial fashion with $30 \mu \mathrm{m}$ resolution. Selected area deposition has also been reported using prepatterned surfaces. For example, it has been demonstrated that charge-patterned insulators can be used to attract a limited amount of oppositely charged particles ${ }^{16,17}$ with $<100 \mathrm{~nm}$ resolution. While prepatterned surfaces provided much higher resolution they remained limited to two-dimensional deposits of a few monolayer thicknesses. The use of prepatterned surfaces had the additional disadvantage that materials could not be altered to vary across the substrate. Very high resolution but monomaterial and two-dimensional prints have been the state of the art using prepatterned surfaces.

Recently Cole et al. ${ }^{18}$ reported a deposition system that eliminated the limitations that the deposits can only be a few monolayers thick by introducing a concept that provides localized charge dissipation. This report extends this printing concept to a multimaterial deposition scheme demonstrating three-dimensional nanostructured deposits that are applied in the combinatory discovery of nanostructured devices. Specifically, we report a multimaterial selected area deposition process that produces $<5 \mathrm{~nm}$ nanoparticles of $\mathrm{Pt}$, $\mathrm{Ag}$, and $\mathrm{W}$, which are subsequently deposited into photoresist openings with sub-100 nm lateral resolutions and aspect ratios of 10 . We demonstrate multimaterial nanostructures that contain sections of $\mathrm{Pt}, \mathrm{Ag}$, and $\mathrm{W}$, whereas individual 
thickness, density, and height is varied and the sequence of the material is switched and selected to be different from one domain to the next. The reported gas phase process shares some of the characteristics of electrodeposition in the liquid phase. The process allows us to control the flux of charged material to locations with $100 \mathrm{~nm}$ lateral resolution to form bridges and interconnects in selected domains or, in the simplest case, to form straight metallic nanowire arrays whose height and density are adjusted to vary across the substrate.

This reported process can be applied to the combinatorial screening of various types of nanostructured deposits for electronic and optoelectronic device applications/discovery. As a first demonstration in this direction, we test whether the tool can be used to identify/discover electrode designs that improve the carrier extraction efficiencies of bulk heterojunction photovoltaic cells. The approach discussed here does not use mechanical shutters to turn ON/OFF deposition in certain areas and provides programmability with much higher resolution and feature density than prior concepts that have been applied to field of optoelectronics and photovoltaics. ${ }^{19-22}$ We explored different interpenetrating electrode designs in particular three-dimensional (3D) nanowire-based electrode arrays where height and density is adjusted from one domain to the next. The protocol varied pitch from 3 to $5 \mu \mathrm{m}$ and height from 500 to $900 \mathrm{~nm}$ to explore the impact on absorption efficiency $\left(\eta_{\AA}\right)$ and minority carrier extraction efficiency $\left(\eta_{\mathrm{ED}}\right)$. The completed solar cell domains share the same substrate, processing, and testing conditions. Short circuit current densities ranged from 7.7 to $12.2 \mathrm{~mA} / \mathrm{cm}^{2}$. The relative power conversion efficiencies (PCE) increased by $47 \%$ in domains that carried dense nanowire arrays when compared to the flat reference on the same substrate.

Results and Discussions. Figure 1 describes the programmable gas phase nanomaterial electrodeposition system where charged material is extracted from the hot plasma region using an externally biased sample that is located in the surrounding low-temperature aerosol region. The system is similar to the original implementation ${ }^{18}$ with the exception that it contains multiple metal electrodes $\mathrm{Pt}$, $\mathrm{Ag}$, and $\mathrm{W}$ (1 $\mathrm{mm}$ rod diameter, Sigma-Aldrich) of the source material instead of a single semiconducting electrode source. The process uses a conventional $<100 \mathrm{~W}$ DC arc discharge where a concentrated plasma surrounds the tip of the consumable cathode. The sample is located in the surrounding aerosol region and carries differently biased domains to attract charged material of a desired polarity. Furthermore, domains are partially screened using a patterned thin layer of Microposit S1805 photoresist, which provides pores and openings to the underlying conducting and externally biased domains. The resist prevents charge dissipation and the material seeks to deposit into the openings. In the illustration, domain 1 is activated first for a certain deposition time of silver (pink color) and a slightly smaller amount of time

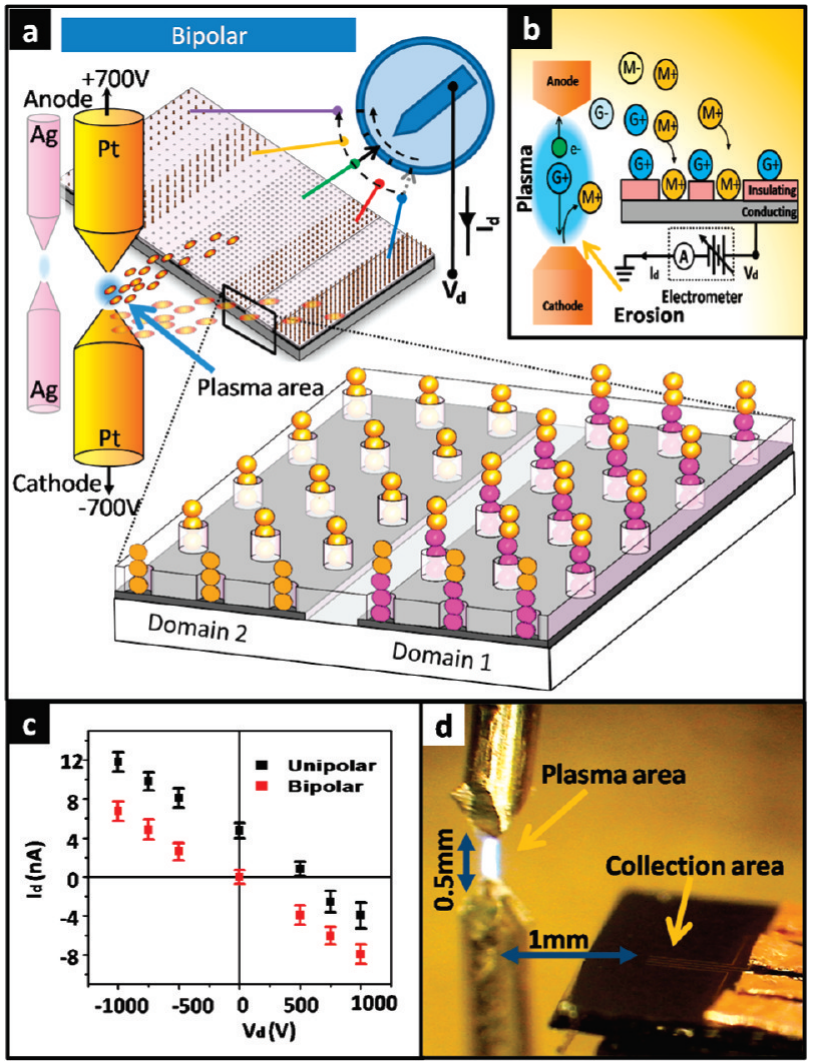

FIGURE 1. Programmable multimaterial deposition process. (a) Schematic depicting a plasma region with two sets of consumable $\mathrm{Ag}$ and Pt electrodes next to a substrate that collects charged nanomaterials using externally biased electrodes to control the material flux and to turn deposition ON/OFF in selected domains. The domains carry a patterned photoresist that act as deposition guides that funnel the material into the openings. (b) Basic concept depicting that charged nanomaterials are produced by cathode erosion and ionization inside the plasma region whereby material flux is diverted toward the biased substrate domains leading to a measurable domain current $I_{\mathrm{d}} ; I_{\mathrm{d}}$ reflects the rate of charge deposition and dissipation on a selected domain. (c) Relationship between the current $I_{\mathrm{d}}$ and domain voltage $V_{\mathrm{d}}$ for bipolar $(+700,-700 \mathrm{~V})$ and unipolar $(+1400 \mathrm{~V}, 0 \mathrm{~V})$ biased arc discharge electrodes (discussed in text). (d) Photograph with physical dimension.

in the deposition cycle of platinum (orange color) leading to the respective film thicknesses. Domain 2 is turned ON in the third deposition cycle to receive a platinum layer (orange) as the first layer. No shutters are involved.

Figure $1 \mathrm{~b}$ depicts the current understanding of the process of (i) particle generation, (ii) charging, and (iii) selected area deposition. (i) The process of particle formation through cathode erosion using atmospheric pressure arc discharge is well established. ${ }^{23-25}$ Electrons generated in the plasma region are accelerated by the applied electric field to the anode, producing gas ions $(\mathrm{G}+$ ) through impact ionization. The gas ions $(\mathrm{G}+)$ move and impact the cathode that leads to cathode erosion. (ii) The eroded material (M) leaves the cathode that is surrounded by a positive $(\mathrm{G}+)$ space charge region and picks up positive charge resulting in the depicted $(\mathrm{M}+)$ particle; negative and neutral particles will be present as well. (iii) Selected area deposition is possible since all 
charged species are influenced by nearby electrodes. Depicted is the case for a negatively biased $\left(V_{\text {sub }}<0\right)$ domain (Figure 1b). The deposition process should be considered as an electrodynamic process since the field distribution evolves over time. Mass and electrical mobility of the involved species is important. The depicted gas ions (G+) have a higher electrical mobility than the heavier metal particle. On a time scale and in the initial stages of the experiments the gas ions $(\mathrm{G}+)$ arrive at the sample surface first, which results in the depicted sheath of space charge $(\mathrm{G}+)$ on the sample surface and alters the potential distribution. Under equilibrium conditions a flux of positive material is established which flows to the conducting and bare electrode sites. The resulting electrical domain current, $I_{\mathrm{d}}$ is recorded with an electrometer (Keithley, model 6517A).

Figure $1 \mathrm{c}$ depicts a graph of the recorded domain current $I_{\mathrm{d}}$ as a function of the domain voltage $V_{\mathrm{d}}$ which is applied by the electrometer to remove the charged species from the gaseous environment. The graph depicts the results of the ionic currents and particle flux for two different types of discharges: (i) bipolar (+700 V upper electrode, $-700 \mathrm{~V}$ lower electrode) and (ii) unipolar (+1400 V, $0 \mathrm{~V}$ ). We choose to use the bipolar case as it provides better control to turn $\mathrm{ON}$ and OFF deposition in certain areas than the unipolar scheme that we used initially. The bipolar discharge has the advantage that grounded substrate domains $V_{d}=0 \mathrm{~V}$ yield zero deposition current which is not the case for the unipolar scheme where a positive domain voltage is required to block deposition.

The dimension of the implementation is shown in the photograph (Figure 1d). The electrodes are enclosed inside an $8000 \mathrm{~cm}^{3}$ plastic polypropylene chamber that allows the control of the pressure and gaseous environment. The reported results here focused on $\mathrm{Pt}, \mathrm{Ag}$, and $\mathrm{W}$, which were found not to be as prone to oxidation as previous experiments that worked with $\mathrm{Si}$ and Ge where specialty gases had to be used. Instead all deposition experiments were performed under a relaxed air environment $\left(78 \% \mathrm{~N}_{2}\right.$ and $\left.21 \% \mathrm{O}_{2}\right)$ at atmospheric pressure.

Figure 2 depicts results of localized deposition of various amounts (500 nm to $2 \mu \mathrm{m}$ ) of Ag, Pt, and W where material type, height, and structure is adjusted from one domain to the next. Figure $2 \mathrm{a}$ depicts different amounts of silver that have been deposited onto selected domains in a two-step sequence. Selection is done by applying $-150 \mathrm{~V}$ to $1 \mu \mathrm{m}$ wide conductive copper domains that run underneath the visible $1 \mu \mathrm{m}$ diameter and $3 \mu \mathrm{m}$ pitched photoresist (Microposit S1805) hole pattern. The deposition times on the left and right were 30 and $60 \mathrm{~s}$, respectively. The heights were approximately 550 and $1100 \mathrm{~nm}$ yielding a deposition rate of $18 \mathrm{~nm} / \mathrm{s}$. This rate is a factor of $20-200$ higher than what is achieved in conventional vacuum thermal or e-beam evaporation systems where $0.1-1 \mathrm{~nm} / \mathrm{s}$ is typical. For $1 \mu \mathrm{m}$ diameter openings, incoming material is funneled into a region that is approximately 10 times smaller than the

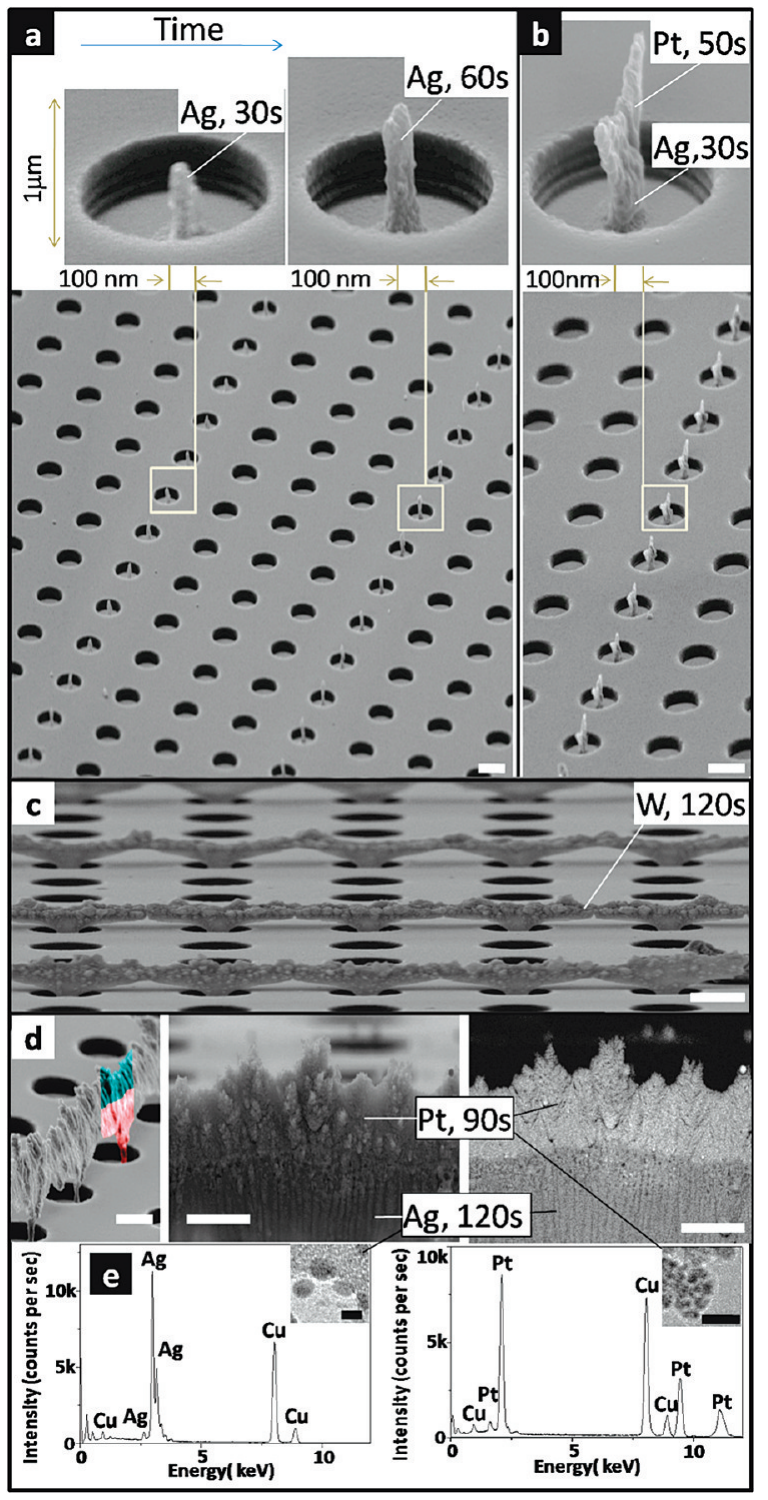

FIGURE 2. Results of multiheight ( $500 \mathrm{~nm}, 1 \mu \mathrm{m}, 2 \mu \mathrm{m})$ and multimaterial (Ag, Pt, and W) towers and bridge arrays. (a) Lines of Ag deposits on doubling the height from one line (30 s) to the next $(60 \mathrm{~s})$ with three empty domains in between. Domain pitch is $3 \mu \mathrm{m}$. Nanolens effect is visible where the material is funneled into the center of the $1 \mu \mathrm{m}$ wide opening. (b) The $30 \mathrm{~s}$ Ag followed by $50 \mathrm{~s}$ Pt depicting the onset of forking to nearest neighbors. (c) the $120 \mathrm{~s}$ $\mathrm{W}$ bridging to nearest ( $3 \mu \mathrm{m}$ pitch) neighbor instead of farthest ( 9 $\mu \mathrm{m}$ pitch) neighbor. (d) Bilayer bridges containing $\mathrm{Pt}$ and $\mathrm{Ag}$ as confirmed by EDX with a primary particle size that is typically smaller than $<5 \mathrm{~nm}$ of $\mathrm{Ag}$ and $<2 \mathrm{~nm}$ for Pt as confirmed by TEM. The interface is visible in the backscattered electron microscope image to the right. One micrometer scale bars in $(a-d), 5 \mathrm{~nm}$ scale bars in the TEM inserts.

opening itself with a positional accuracy of 20-30 nm (STD). The positional accuracy is a relative alignment accuracy with respect to the given photoresist structure; absolute alignment accuracy remains limited by the mask aligner. Resulting deposits grow into well-defined needles/towers with a diameter of about $100 \mathrm{~nm}$. The insulating surface cannot dissipate charge and prevents the deposition of material, 
which is visible in the figures; no particles are found on the photoresist itself. In fact, the incoming positive material maintains a distance away from the photoresist, which requires a potential landscape or potential trough that favors the material to follow down a $100 \mathrm{~nm}$ thin channel to the center of the photoresist opening in the illustrated case. This means that the insulator floats up to a more positive potential in the initial stage of the experiments, which is maintained under equilibrium conditions and is utilized here to form nanolense arrays. Incoming material deposits on top of the advancing structures and maintains the cylindrical nanolens symmetry well above the surface of the $500 \mathrm{~nm}$ deep resist openings before they begin to spread out and change shape. Structures maintain the shape if the deposition process is stopped before they grow much taller than the photoresist film thickness itself; much taller structures will change shape similar to the "mushrooming" effect in electrodeposition in the liquid phase which is shown in the Supporting Information Figure S1.

While it is more difficult to predict the shape under prolonged deposition the structures remain well-defined. This is illustrated in Figure $2 \mathrm{~b}$ where we have selected the deposition of two different materials. The structures contain about $500 \mathrm{~nm}$ of Ag and $800 \mathrm{~nm}$ of Pt and show the onset of forking to nearest neighbors. Forking to nearest neighbors is due to the collective behavior of the underlying electrode, photoresist pattern and emerging nanostructures. Both far and near fields need to be considered to understand the forking behavior. At a distance that is sufficiently large, the underlying negatively biased holes are seen as a negative line, which concentrates incoming material into a twodimensional (2D) sheath that is directed toward the surface. In close proximity, the $2 \mathrm{D}$ sheath separates into isolated flux channels until they reach the surface of the emerging nanostructures.

The separation into isolated flux channels leads to the observed forking and ultimately the formation of interconnects between nearest neighbors. This is illustrated in Figure $2 \mathrm{c}$ where the concept is used to form interconnects between nearest neighbors using a third material, tungsten, and $120 \mathrm{~s}$ deposition. Figure $2 \mathrm{~d}$ repeats this experiment forming a stack of two different materials, $120 \mathrm{~s}$ of silver and $90 \mathrm{~s}$ of platinum. These are tilted views of the sample. The green and pink colors were added to help distinguish the different domains. The interface is well resolved in the backscattered electron image (BSE), which is sensitive to the atomic number difference of the materials; those with higher atomic numbers (Pt in the shown case) appear brighter. Figure $2 \mathrm{e}$ contains material-specific data of the materials that are formed using the arc discharge process. Specifically, we collected some of the material on a copper transmission electron microscope (TEM) grid and analyzed the samples in a TEM (Phillips FEI T12). The inserted TEM image depicts $5 \mathrm{~nm}$ silver and $2 \mathrm{~nm}$ platinum particles. The particles are typically smaller than $5 \mathrm{~nm}$ and are confirmed to be Ag and
Pt using energy dispersive X-ray spectroscopy (EDS). The TEM image and EDS for tungsten are included in the Supporting Information.

Figure 3 provides results where the programmable gas phase deposition tool has been applied to the general idea of combinatorial screening of nanostructured deposits for electronic and optoelectronic device applications. The specific demonstration focuses on the identification of nanostructured electrode designs that improve the carrier extraction efficiencies of bulk heterojunction photovoltaic cells. The p-n junction where electron-hole pairs are separated is no longer planar in these devices and the electrical contacts and geometry is one of the most critical elements. The use of nanostructured 3D electrodes has been reported previously to improve device efficiency. ${ }^{26}$ However, little is known how the density and height variations impact the device performance. The current practice of processing separate substrates in different runs often yields inconclusive answers. To overcome this challenge of processing different substrates we deposited domains of nanowire arrays with different heights and density on a single substrate by controlling the bias levels in the different domains. The protocol varied nanowire pitch from 3 to $5 \mu \mathrm{m}$ and height from 500 to $900 \mathrm{~nm}$ from one domain to the next. Short circuit current densities $\left(J_{\mathrm{sc}}\right)$ ranged from 7.7 to $12.2 \mathrm{~mA} /$ $\mathrm{cm}^{2}$. The power conversion efficiencies (PCE) increased by $47 \%$ in domains that carried dense nanowire arrays when compared to the flat reference topology on the same substrate.

Figure 3 a illustrates the procedure and the results whereby the substrate was divided into five separate domains. Individual domains were formed using $500 \mu \mathrm{m}$ wide, $2 \mathrm{~cm}$ long, and $150 \mathrm{~nm}$ thick ITO regions that are located underneath a $500 \mathrm{~nm}$ thick Microposit S1 805 photoresist. The resist was patterned to provide $1 \mu \mathrm{m}$ diameter openings to the ITO domains. The pitch was $5 \mu \mathrm{m}$ on the left (domains 1 and 2) and $3 \mu \mathrm{m}$ on the right (domains 4 and 5). Domain 3 served as a reference and was not patterned. We first connected domain 1 to the negative bias and grounded the other four ITO domains to prevent deposition in undesired areas. We followed the same procedure on domains $2-5$. The deposition times were 40,60, 0, 40, and $60 \mathrm{~s}$; deposition material was platinum in each case. The SEMs $\left(a_{1}-a_{5}\right)$ depict the resulting $80-150 \mathrm{~nm}$ diameter deposits whereby the height varied from left to right. Specific height values were 600 , $900,0,500$, and $800 \mathrm{~nm}$. The histogram in Supporting Information Figure S3 depicts the measured variation in height in domain 5. The standard deviation (STD) was less than $13.1 \mathrm{~nm}$ for the $800 \mathrm{~nm}$ tall structures. Supporting Information Figure S4 provides a larger area picture to illustrate the uniformity.

It shows the processing steps and the respective SEMs that are required to complete the devices in Supporting Information Figure S5. The steps are (1) nanowire deposition using photoresist guides and bias control, (2) removal of the 

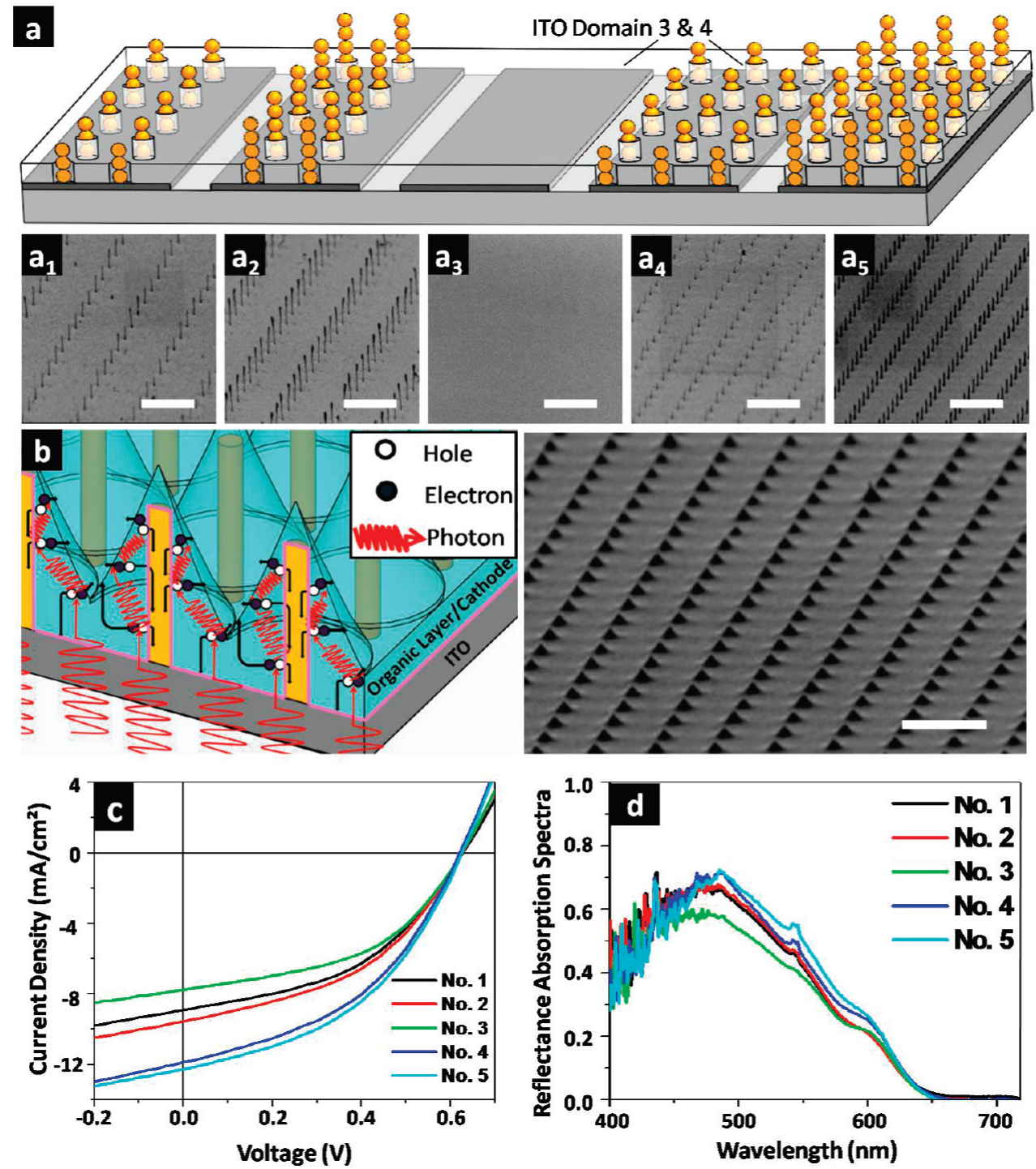

FIGURE 3. Combinatorial screening of nanostructured Pt nanowire electrodes adjusting pitch and height impacting carrier extraction and absorption efficiencies in organic P3HT-PCBM bulk heterojunction photovoltaic cells. (a) Schematic using photoresist guides to define circular opening using five different domains to receive $600,900,0,500$, and $800 \mathrm{~nm}$ of Pt; the pitch is $5 \mu \mathrm{m}$ on the left (a1), (a2) and $3 \mu \mathrm{m}$ (a3), (a4) on the right. (b) Graphical representation of the completed structures depicting nanowire anodes that penetrate the organic layer next to the SEM image of the P3HT-PCBM bulk heterojunction photovoltaic cell on domain 4; scattering is depicted to take place at the embedded Pt nanowires and the conical Al coated top surface. (c,d) $I / V$ curves and reflectance absorption spectra of the different domains. Five micrometer scale bars in (a1-a5) and the inset of (b).

photoresist guides using an oxygen plasma, (3) spin coating of the electron blocking layer (PEDOT-PSS), active blend layer (P3HT-PCBM), and (4) vapor deposition of lithium fluoride (LiF) tunneling layer and aluminum top contact. The graphical representation in Figure 3b shows the 3-dimensional cells and its basic operation. The film thickness and energy level diagram of the used materials are included in Supporting Information Figure S5. The structure is illuminated from the bottom and electron hole pairs are produced in the active blend layer and separated by the built-in potential between the HOMO of the P3HT donor and the LUMO of the PCBM acceptor material. ${ }^{27}$ Holes are collected by the Pt nanostructures and the ITO substrate while electrons are collected at the LiF/Al conical contacts. The graphi- cal representation illustrates wide angle photon scattering due to the curvature of the top LiF/Al reflective contact and bottom nanowire contact where scattering takes place. Wide angle scattering and light trapping is used in the following section to explain the observed measurement results.

Figure $3 \mathrm{c}$ depicts the recorded current-voltage characteristics. The calculated device parameters are summarized in Table 1. The tabulated fill factor FF represents the ratio between the measured maximum output power normalized by the product of recorded open circuit voltage $V_{\text {oc }}$ and recorded short circuit current $I_{\mathrm{sc}}$. The active area of each domain was $0.02 \mathrm{~cm}^{2}$ that was determined using the dimensions of the intersecting $500 \mu \mathrm{m}$ wide ITO bottom and $4 \mathrm{~mm}$ wide aluminum top contact. Domains with Pt tower deposits 
TABLE 1. Device Performance in the Respective Domains

\begin{tabular}{cccccc}
\hline domain & $V_{\mathrm{oc}}[\mathrm{V}]$ & $J_{\mathrm{sc}}\left[\mathrm{mA} / \mathrm{cm}^{2}\right]$ & $\mathrm{FF}[\%]$ & $\mathrm{PCE}[\%]$ & $\begin{array}{c}\text { relative PCE } \\
\text { changes [\%] }\end{array}$ \\
\hline no. 1 & 0.631 & 8.92 & 43.9 & 2.47 & 9.29 \\
no. 2 & 0.627 & 9.57 & 43.4 & 2.61 & 15.4 \\
no. 3 & 0.623 & 7.76 & 46.8 & 2.26 & 0 \\
no. 4 & 0.621 & 11.88 & 43.1 & 3.18 & 40.7 \\
no.5 & 0.626 & 12.28 & 43.4 & 3.34 & 47.7
\end{tabular}

performed better than the flat reference domain No. 3. The relative power conversion efficiency was enhanced by 9.29 , $15.4,40.7$, and $47.7 \%$ for domains $1,2,4$, and 5 , respectively, when compared to the flat reference domain 3 . Most notably, the nanowire deposits have a strong effect on the recorded short circuit current and almost no effect on the open circuit voltage. Dense arrays in domain 4 and 5 produced higher currents $\left(11.9-12.3 \mathrm{~mA} / \mathrm{cm}^{2}\right)$ than the less dense arrays in domain 1 and $2\left(8.9-9.6 \mathrm{~mA} / \mathrm{cm}^{2}\right)$ or the flat reference $\left(7.8 \mathrm{~mA} / \mathrm{cm}^{2}\right)$. Increasing the height of the towers from 500 to $900 \mathrm{~nm}$ improved the devices as well but at a lesser rate than doubling the density. We also tested wires that were $>1.5 \mu \mathrm{m}$ long and found these to work less favorable; this was attributed to the onset of shorts since some of the wires had an insufficiently thin coating at their top. Five experiments on separate substrates all found domain 5 to have the highest performance. Figure $3 \mathrm{~d}$ shows the optical absorption spectra, which suggest that the improvement of the device is at least in part due to an increased absorption. The spectra were collected from each domain using a reflection fiber optic micro spectrometer (Ocean Optics, USB4000 VIS-NIR) with a spot size of $400 \mu \mathrm{m}$. For example, at $485 \mathrm{~nm}$ the relative absorption increased by 15 , 17,25 , and $26 \%$ for domains $1,2,4$, and 5 when compared to the flat reference. The absorption spectra of the domains with nanostructures are also slightly broader than without. These results are in agreement with an earlier study where nanostructured topologies increased light trapping and adsorption. ${ }^{28,29}$ The increased optical absorption alone (up to $26 \%$ ), however, is insufficient to explain the relative increase in efficiency (up to $48 \%$ ) for the dense nanowire arrays when compared with the flat reference domain. This mismatch can be explained by the fact that penetrating 3D electrodes are also known to improve electron-hole pairs separation and collection. ${ }^{30}$ Like conventional nanowirebased architectures our 3D nanostructured electrodes shares this general idea and should provide a greater degree of freedom for the collection of holes at the Pt towers and electrons at the Al top contact. The combination of the two mechanisms, enhancement of light absorption and improvement of carrier collection, is likely the cause for improvement of the short circuit currents in the different domains. Despite the improvements, the reported power conversion efficiencies remain lower than the current record..$^{31-33}$ Our devices are not fabricated under the exclusion of oxygen, which is known to limit the efficiency to the reported values. For example, domain 5 short circuit current drops by $58 \%$ after $10 \mathrm{~h}$ of air exposure, which is a common problem for organic thin film solar cells. The exposure to air is presently limited to $10 \mathrm{~min}$ but is not completely eliminated since the characterization and evaporation tool are in physically different locations. The strength of the combinatorial approach however remains. It provides many different domains and a flat reference on a single substrate, which provides a more reliable comparison since all domains are exposed to the same testing and environmental conditions.

Conclusions. In conclusion, the developed process can be used to form charged, $<5 \mathrm{~nm}$ sized particles (silver, tungsten, and platinum) at atmospheric pressure. The material can be deposited to form thick $500 \mathrm{~nm}$ to $2 \mu \mathrm{m}$ nanostructured deposits on selected domains. The sequence and amount of material can be mixed and matched yielding multimaterial deposits like bridges, towers, interconnects, and nanowires where height, density, and material composition can be adjusted from one domain to the next. We anticipate that the process can be extended to other metals as well as semiconductors. Here we used an oxygencontaining environment to deposit metallic material that did not oxidize readily. To extend the atmospheric pressure process to fabricate nonoxidized semiconducting nanostructured deposits is likely going to require a transition to an oxygen-free environment. Little is known about atmospheric pressure deposition systems, which appear to have many distinguishing features when compared to vacuum vapor phase deposition systems. This study reveals that the deposition rate can be at least a factor of 10 higher. The material can be deposited onto desired locations with a minimal lateral resolution of $50-100 \mathrm{~nm}$ and a positional accuracy of 20-30 nm (STD). No liftoff is required and less material is lost as a result. The overall cost to build this system is less than $\$ 1,000$ since it operates at atmospheric pressure. The point source used, however, has some limitation with respect to uniformity if one considers larger $\left(>1 \mathrm{~cm}^{2}\right)$ samples. This could be overcome by extending the process to tip arrays or orbiting substrates, which has not yet been tested. Instead, we decided to apply the method to identify in a combinatorial way 3D nanostructured electrode designs that improve light scattering, absorption, and minority carrier extraction of 3D bulk heterojunction photovoltaic cells. Photovoltaic cells from domains with long and dense interpenetrating nanowire arrays improve the relative power conversion efficiency by $47 \%$ when compared to flat domains on the same substrate. We have not yet tested multiple parameters at a time but it should be possible to test other designs where the material is varied from one domain to the next, or where structures are formed that do not simply change the pitch or height. While the interpretation of the results would be more involved such an approach could lead to the discovery of new higher performance architectures. 
Acknowledgment. The authors acknowledge support of this work by NSF DMI-0755995, NSF DMI-0621137, and NSF DMI-0556161.

Supporting Information Available. Experimental methods, additional figures, and references. This material is available free of charge via the Internet at http://pubs.acs. org.

\section{REFERENCES AND NOTES}

(1) Taton, T.; Mirkin, C.; Letsinger, R. Science 2000, 289, 1757-1760.

(2) Jacobs, H.; Campbell, S.; Steward, M. Adv. Mater. 2002, 14, $1553-$ 1557.

(3) Liu, W. J. Biosci. Bioeng. 2006, 102, 1-7.

(4) Pavesi, L.; Negro, L.; Mazzoleni, C.; Franzo, G.; Priolo, F. Nature 2000, 408, 440-444.

(5) McDonald, S.; Konstantatos, G.; Zhang, S.; Cyr, P.; Klem, E.; Levina, L.; Sargent, E. Nat. Mater. 2005, 4, 138-142.

(6) Pan, Z.; Dai, Z.; Wang, Z. Science 2001, 291, 1947-1949.

(7) Feldman, Y.; Wasserman, E.; Srolovitz, D.; Tenne, R. Science 1995, 267, 222-225

(8) Huang, M.; Wu, Y.; Feick, H.; Tran, N.; Weber, E.; Yang, P. Adv. Mater. 2001, 13, 113-116.

(9) Morales, A.; Lieber, C. Science 1998, 279, 208-211.

(10) Facsko, S.; Dekorsy, T.; Koerdt, C.; Trappe, C.; Kurz, H.; Vogt, A.; Hartnagel, H. Science 1999, 285, 1551-1553.

(11) Iijima, S. Nature 1991, 354, 56-58.

(12) Schwyn, S.; Garwin, E.; Schmidt-Ott, A. J. Aerosol Sci. 1988, 19 , 639-642.

(13) Camata, R.; Atwater, H.; Vahala, K.; Flagan, R. Appl. Phys. Lett. 1996, 68, 3162-3164.

(14) Akhatov, I.; Hoey, J.; Swenson, O.; Schulz, D. J. Aerosol Sci. 2008 39 (8), 691-709.

(15) Di Fonzo, F.; Gidwani, A.; Fan, M.; Neumann, D.; Iordanoglou, D.; Heberlein, J.; McMurry, P.; Girshick, S.; Tymiak, N.; Gerberich, W. Appl. Phys. Lett. 2000, 77, 910.
(16) Barry, C.; Steward, M.; Lwin, N.; Jacobs, H. Nanotechnology 2003, 14,1057

(17) Zonnevylle, A.; Hagen, C.; Kruit, P.; Schmidt-Ott, A. Microelectron. Eng. 2009, 86, 803-805.

(18) Cole, J.; Lin, E.; Barry, C.; Jacobs, H. Appl. Phys. Lett. 2009, 95, 113101

(19) Schmitz, C.; Thelakkat, M.; Schmidt, H. Adv. Mater. 1999, 11, $821-826$

(20) Sun, T.; Jabbour, G. MRS Bull. 2002, 27, 309-315.

(21) Godovsky, D.; Chen, L.; Pettersson, L.; Inganäs, O.; Andersson, M.; Hummelen, J. Advanced Materials fo Optics and Electronics 2000, 10, 47-54.

(22) Haber, J.; Gerein, N.; Hatchard, T.; Versavel, M. In Combinatorial Discovery of New Thin Film Photovoltaics, 31 st IEEE Photovoltaic Specialists Conference, Lake Buena Vista, FL, 2005; Institute for Electrical and Electronics Engineers; pp 155-158.

(23) Aleksandrov, N.; Bazelyan, E. J. Phys. D: Appl. Phys. 1996, 29, $740-752$.

(24) Aleksandrov, N.; Bazelyan, E. Plasma Sources Sci. Technol. 1999, 8, 285-294.

(25) Rodrfguez, A.; Morgan, W.; Touryan, K.; Moeny, W.; Martin, T. J. Appl. Phys. 1991, 70, 2015-2022.

(26) Mor, G.; Shankar, K.; Paulose, M.; Varghese, O.; Grimes, C. Appl. Phys. Lett. 2007, 91, 152111

(27) Brabec, C. J.; Cravino, A.; Meissner, D.; Sariciftci, N. S.; Fromherz, T.; Rispens, M. T.; Sanchez, L.; Hummelen, J. C. Adv. Funct. Mater. $2001,11,374-380$.

(28) Roman, L.; Inganas, O.; Granlund, T.; Nyberg, T.; Svensson, M.; Andersson, M.; Hummelen, J. Adv. Mater. 2000, 12, 189-195.

(29) Na, S.; Kim, S.; Kwon, S.; Jo, J.; Kim, J.; Lee, T.; Kim, D. Appl. Phys. Lett. 2007, 91, 173509

(30) Kayes, B.; Atwater, H.; Lewis, N. J. Appl. Phys. 2005, 97, 114302

(31) Ma, W.; Yang, C.; Gong, X.; Lee, K.; Heeger, A. Adv. Funct. Mater. 2005, 15, 1617-1622.

(32) Park, S.; Roy, A.; Beaupré, S.; Cho, S.; Coates, N.; Moon, J.; Moses, D.; Leclerc, M.; Lee, K.; Heeger, A. Nat. Photonics 2009, 3, 297 302

(33) Reyes-Reyes, M.; Kim, K.; Dewald, J.; Lopez-Sandoval, R.; Avadhanula, A.; Curran, S.; Carroll, D. Org. Lett. 2005, 7, 5749-5752. 